\title{
Register Forschungsförderung der Deutschen Gesellschaft der Plastischen, Rekonstruktiven und Ästhetischen Chirurgen (DGPRÄC) und Forschungsförderungs-Bericht 2017/2018
}

\section{Registry Research Funding of the German Society of Plastic, Reconstructive and Aesthetic Surgeons (DGPRÄC) and Research Funding Report 2017/2018}

Autoren

R. E. Giunta ${ }^{1}{ }^{*}$, N. Möllhoff ${ }^{*}$, R. E. Horch ${ }^{2}$, L. Prantl ${ }^{3}$, J. P. Beier ${ }^{4}$, A. Daigeler ${ }^{5}$, A. Dragu ${ }^{6}$, S. U. Eisenhardt' ${ }^{7}$ P. C. Fuchs ${ }^{8}$,

G. Germann ${ }^{9}$, T. Hirsch ${ }^{10}$, M. Infanger ${ }^{11}$, R. Jakubietz ${ }^{12}$, U. Kneser ${ }^{13,14}$, S. Langer ${ }^{15}$, M. Lehnhardt ${ }^{16}$, H. G. Machens ${ }^{17}$,

P. Mailänder ${ }^{18}$, B. Reichert ${ }^{19}$, C. Radtke $^{20}$, D. J. Schaefer ${ }^{21}$, F. Siemers 22 , G. B. Stark ${ }^{7}$, H. U. Steinau ${ }^{23}$, P. M. Vogt ${ }^{24}$

Institute

1 Abteilung für Handchirurgie, Plastische Chirurgie und Ästhetische Chirurgie, Klinikum der Ludwig-Maximilians Universität München

2 Plastisch- und Handchirurgische Klinik, Universitätsklinikum Erlangen, Friedrich-Alexander-Universität Erlangen-Nürnberg FAU

3 Hochschulzentrum für Plastische und Ästhetische, Hand- und Wiederherstellungschirurgie der Universität Regensburg

4 Klinik für Plastische Chirurgie, Hand- und Verbrennungschirurgie, Universitätsklinikum RWTH Aachen

5 Plastische Chirurgie, Hand-, Rekonstruktive und Verbrennungschirurgie an der Eberhard-Karls Universität/BC Unfallklinik Tübingen

6 Abteilung für Plastische und Handchirurgie, Universitätsklinikum Carl Gustav Carus, Dresden

7 Klinik für Plastische und Handchirurgie, Medizinische Fakultät, Universitätsklinikum Freiburg

8 Klinik für Plastische und Ästhetische Chirurgie, Handchirurgie - Schwerbrandverletztenzentrum -, Klinikum Köln-Merheim

9 ETHIANUM, Klinik für Plastische, Ästhetische und Präventive Medizin am Universitätsklinikum Heidelberg

10 Plastische-, Rekonstruktive und Ästhetische Chirurgie, Fachklinik Hornheide, Plastische Chirurgie, Universitätsklinikum Münster

11 Klinik für Plastische, Wiederherstellungs-und Handchirurgie, Otto-von-Guericke-Universität, Magdeburg

12 Klinik für Unfall-, Hand-, Plastische und Wiederherstellungschirurgie/Sektion Plastische \& Ästhetische Chirurgie/Universitätsklinikum Würzburg

13 Klinik für Hand-, Plastische \& Rekonstruktive Chirurgie/ Schwerbrandverletztenzentrum, BG-Unfallklinik Ludwigshafen

14 Klinik für Plastische und Handchirurgie, Ruprechts Karls Universität Heidelberg

15 Abteilung für Plastische-, Ästhetische- und spezielle Handchirurgie, Universitätsklinikum Leipzig - AöR
16 Klinik für Plastische Chirurgie und Schwerbrandverletzte, BG-Universitätsklinik Bergmannsheil

17 Klinik und Poliklinik für Plastische Chirurgie und Handchirurgie/Klinikum rechts der Isar, Technische Universität München

18 Abteilung für Plastische Chirurgie, Handchirurgie, Zentrum für Schwerbrandverletzte, Universitätsklinikum SchleswigHolstein, Campus Lübeck

19 Klinik für Plastische, Wiederherstellende und Handchirurgie, Zentrum für Schwerbrandverletzte, Klinikum Nürnberg-Süd, Universitätsklinik der Paracelsus Medizinischen Privatuniversität

20 Klinische Abteilung für Plastische und Rekonstruktive Chirurgie, Universitätsklinik für Chirurgie

21 Plastische, Rekonstruktive, Ästhetische und Handchirurgie, Universitätsspital Basel

22 Klinik für Plastische Chirurgie und Handchirurgie, Brandverletztenzentrum, Berufsgenossenschaftliche Kliniken Bergmannstrost, Martin-Luther Universität Halle-Wittenberg

23 Universitätsklinik für Unfallchirurgie / Orthopädie, Plastische und Rekonstruktive Chirurgie Schwerpunkt Sarkomchirurgie, WTZ

24 Klinik und Poliklinik für Plastische, Hand- und Wiederherstellungschirurgie, Medizinische Hochschule Hannover

Schlüsselwörter

Akademische Plastische Chirurgie, Forschungsförderung, DFG, DGUV, BMBF, BMWi, Peer Review, Forschungsförderungsbericht

Key words

Academic plastic surgery, research funding, DFG, BMBF, DGUV, BMWi, peer review, research funding report

eingereicht 27.09.2018

akzeptiert $\quad 01.10 .2018$

*equally contributed to the paper 
Bibliografie

DOI https://doi.org/10.1055/a-0770-3485

Handchir Mikrochir Plast Chir 2018; 50: 414-421

(c) Georg Thieme Verlag KG Stuttgart · New York

ISSN 0722-1819

Korrespondenzadresse

Univ.-Prof. Dr. med. Riccardo Giunta

Abteilung für Handchirurgie, Plastische Chirurgie und Äs-

thetische Chirurgie

Klinikum der Ludwig-Maximilians Universität München

Pettenkoferstr. 8a, 80336 München

Email r.giunta@med.uni-muenchen.de

\section{ZUSAMMENFASSUNG}

Hintergrund Dieser Bericht baut auf dem Forschungsförderungsbericht aus dem Jahr 2015/2016 auf und dient der Bekanntmachung der akademischen Forschungsleistung an Universitätskliniken der Deutschen Gesellschaft der Plastischen, Rekonstruktiven und Ästhetischen Chirurgen (DGPRÄC) für 2017/2018. Berücksichtigt wurden dabei Anträge auf Forschungsförderung bei öffentlichen, nicht-öffentlichen, oder industriellen Förderinstituten. Gleichzeitig soll dieser Beitrag die Anzahl der genehmigten Anträge bei der Deutschen Forschungsgemeinschaft (DFG) aus der Plastischen Chirurgie, Thorax- und Gefäßchirurgie gegenüberstellen. Innerhalb dieser jüngeren selbständigen chirurgischen Fachgebiete wird das seit 1993 eigenständige Fachgebiet Plastische Chirurgie in der Fächerstruktur der DFG Fachkollegien immer noch nicht separat geführt, sondern dem Fachkollegium Orthopädie und Unfallchirurgie zugeordnet. Dies führt dazu, dass die Anträge nicht fachspezifisch begutachtet werden.

Material und Methoden Die bereits etablierte online Datenbank (https://docs.google.com/forms/d/1OaSnHyKTysawil1ie7kfUxDf7nJP_RiTUJTsnb7Mq_E/edit ) zur Meldung beantragter/genehmigter und abgelehnter Forschungsförderungen öffentlicher, nicht-öffentlicher und industrieller Förderinstitutionen wurde fortgeführt und gemeinsam mit den Anträgen aus der öffentlichen Datenbank der DFG, das Geförderte Projekte Informationssystem (GEPRIS), ausgewertet. Ergebnisse Im Vergleich zum letzten Beobachtungszeitraum von 2015/2016 nahm die Anzahl genehmigter Anträge aus öffentlichen Einrichtungen (DFG, BMBF, BMWi, EU) von 23 auf 27 zu. Aus den chirurgischen Fachgebieten Thorax- und Gefäßchirurgie waren jeweils 9 bzw. 8 DFG
Anträge im GEPRIS dokumentiert, wohingegen 19 Bewilligungen von Anträgen der Plastischen Chirurgie identifiziert wurden.

Schlussfolgerung Durch die vorliegende Auswertung konnten wir zeigen, dass das Aufkommen an Anträgen aus dem Fachgebiet der Plastischen Chirurgie denen der anderen eigenständig geführten Fachgebiete entspricht, wobei sogar eine höhere Anzahl zu verzeichnen ist. Vor diesem Hintergrund ist die gegenwärtige Subsummierung seitens der DFG, auch im Hinblick auf eine öffentlich nachvollziehbare Vergabe der Fördersummen, nicht zu akzeptieren.

\section{ABSTRACT}

Introduction This report serves to publicize the research of academic institutions for Plastic Surgery within our society DGPRÄC in 2017/2018 and sequels the funding report of 2015/2016. Applications to public, non-public, or industrial funding organizations were evaluated. At the same time, this paper analyses the number of approved DFG applications in Plastic, Thoracic and Vascular Surgery in the GEPRIS system. Contrary to these specialties, Plastic Surgery is not classified as an independent speciality in the subject structure of the DFG review board which results in a lack of transparency concerning Plastic Surgery research work.

Materials and Methods Our previously established online database (https://docs.google.com/forms/d/10aSnHyKTysawil1ie7kfUxDf7nJP_RiTUJTsnb7Mq_E/edit ) for reporting requested/ approved and rejected research applications to public, non-public and industrial funding organizations was continued and evaluated together with applications found in the DFG's public database GEPRIS.

Results Compared to the previous year's report, the number of approved applications from public research organizations (DFG, BMBF, BMWi, EU) was increased from 23 to 27 . We identified 19 approved DFG applications from Plastic Surgery, as compared to 9 and 8 applications by Thoracic and Vascular Surgery, respectively.

Summary Taken together, this data emphasizes that our research is at least equal to that of other newly established surgical specialties within the framework of the DFG. Accordingly, we hope to provide further arguments for an adaptation of the DFG review boards subject structure to include Plastic Surgery as an independent specialty as it is for Vascular Surgery and Thoracic Surgery.

\section{Einleitung}

Im Jahr 2016 wurde zur Verbesserung der Sichtbarkeit der Forschungsleistung unseres Fachgebiets die erste Auswertung bewilligter Forschungsförderung öffentlicher Fördereinrichtungen (DFG, BMBF, BMWi, EU) nach Standorten der Universitätsklinika erstellt [1]. Zudem richteten wir eine öffentlich verfügbare Internetplattform ein (https://docs.google.com/forms/d/1OaSnHyKTysawil1ie7kfUxDf7nJP_RiTUJTsnb7Mq_E/edit), die die Dokumentati- on der Anträge bereits bei Antragstellung ermöglicht. Diese Arbeit entspricht dem Forschungsförderungs-Bericht der Plastischen Chirurgie für das Jahr 2017/2018.

Aktuell wird die Plastische Chirurgie in der Organisationsstruktur der Universitätskliniken durch 11 eigenständige Kliniken, sowie 12 Abteilungen und Sektionen repräsentiert. 13 universitäre Standorte weisen bislang keine eigenständige Organisationsstruktur des Fachgebietes Plastische Chirurgie auf. Diese wird in diesen Fällen in 
Form eines Oberarztes mit Zuordnung zu einem anderen chirurgischen Fachgebiet abgebildet. Das Fachgebiet der Plastischen Chirurgie im Sinne eigenständiger Kliniken mit eigener Forschungsinfrastruktur an deutschen Universitätsklinika erscheint daher noch unterrepräsentiert und bildet nicht die fortschreitende Spezialisierung in der Chirurgie ab. Auch die Übernahme der Leitung von chirurgischen Kliniken durch Plastische Chirurgen, als interdisziplinäre Spezialisten mit Schnittstellen zu verschiedenen chirurgischen Fachbereichen, ist ein Aspekt der zukünftigen Entwicklung [2]. Kürzlich konnte gezeigt werden, dass sich die Art der Organisationsstruktur bedeutend auf die Publikationsleistung auswirkt. Nachweislich besteht demnach ein direkter Zusammenhang zwischen Grad der Eigenständigkeit und Publikationsleistung, -qualität, Ressourcen und Forschungsinfrastruktur [3-5].

Anderen kleineren chirurgischen Fachgebieten, wie etwa der Thoraxchirurgie oder Gefäßchirurgie, ergeht es im Hinblick auf die noch bestehende Unterrepresäntation an den Universitätskliniken ähnlich. Obwohl ihre Fachgesellschaften geringere Mitgliederzahlen aufweisen, werden sie jedoch beispielsweise bei DFG Anträgen namentlich im DFG Fachkollegium 205-26 Herz-, Thorax-, Gefäßchirurgie aufgeführt. DFG Anträge aus unserem Fachgebiet hingegen können weiterhin nicht namentlich der Plastischen Chirurgie zugeordnet werden. Stattdessen wird eine Zuordnung zum Fachkollegium Orthopädie und Unfallchirurgie getroffen, welches zukünftig in Orthopädie, Unfallchirurgie, rekonstruktive Chirurgie umbenannt wird. Dieses bildet aber in keiner Weise die wissenschaftlichen Schwerpunkte der vier Säulen der Plastischen Chirurgie und die in hohem Maße interdisziplinären Aktivitäten mit in weiteren Fachkollegien repräsentierten Schwerpunkten (Transplantation, Onkologie, etc) ab. Somit erscheint auch die finale Entscheidung innerhalb des gegenwärtigen Fachkollegiums, dem keine Plastischen Chirurgen angehören, unverständlich.

Durch den Vergleich der bewilligten DFG Anträge der oben genannten Fachgebiete belegen wir mit dieser Arbeit, dass die Anzahl der geförderten Anträge der Plastischen Chirurgie die der Thoraxoder Gefäßchirurgie nicht nur übertrifft, sondern durch die Zuordnung zur Orthopädie und Unfallchirurgie unzureichend abgebildet wird. Der Vergleich liefert somit Argumente, die für eine Anpassung der Fächerstruktur der DFG sprechen.

\section{Methoden}

Wie im Forschungsförderungsbericht 2015/2016 wurden für diese Arbeit Anträge von plastisch-chirurgischen Einrichtungen der Universitätsklinika bei öffentlichen Förderinstitutionen wie DFG, BMBF, BMWi und EU gesammelt und ausgewertet. Dazu wurde die öffentliche Datenbank der DFG, das Geförderte Projekte Informationssystem (GEPRIS) verwendet. [6] Zusätzlich wurden die Angaben der Zuständigen der Universitätskliniken auf der von uns erstellten online Datenbank (https://docs.google.com/forms/d/10aSnHyKTysawil1ie7kfUxDf7nJP_RiTUJTsnb7Mq_E/edit) ausgewertet. Auf dieser baten wir die jeweiligen Leiter der Einrichtungen um Mitteilung der beantragten, in Begutachtung befindlichen, abgelehnten und geförderten Projekte jeglicher öffentlicher, nicht-öffentlicher, industrieller oder anderer Förderinstitute. Im Vergleich zum Forschungsförderungsbericht aus dem Jahr 2015/16 konnten wir so ein noch umfassenderes Bild unserer Forschungsleistung als Fachgebiet erreichen. Um den Vergleich zur Vorjahres Auswertung zu ermöglichen, wurden die öffentlichen Förderanträge (DFG, BMBF, BMWi, EU) gesondert ausgewertet. ( $\triangleright$ Tab. 1 und $\triangleright$ Tab. 2)

Um Thorax- und Gefäßchirurgische DFG Anträge der Universitätsklinika zu identifizieren und vergleichend zur Plastischen Chirurgie darzustellen, wurden folgende Suchbegriffe im Suchportal GEPRIS unter dem Reiter „Institutionen“ verwendet:

„Thorax“, „Thoraxchirurgie“, „Gefäß“, „Gefäßchirurgie“, „Plastisch“, „Plastische Chirurgie“

Wie in $>$ Tab. 3 dargestellt, wurden die jeweiligen Anträge aus den entsprechenden Kliniken anhand ihrer fachlichen Zuordnung

\ Tab. 1 Übersicht über die bewilligte Forschungsförderung öffentlicher Fördereinrichtungen (DFG, BMBF, BMWi und EU) nach Standorten.

\begin{tabular}{|l|l|l|l|l|}
\hline Uniklinik & Art der Förderung & Projekttitel & Zuordnung & Zeitraum \\
\hline Aachen & DFG Sachbeihilfe & $\begin{array}{l}\text { Evaluierung eines neuen Ansatzes für die Weichteilgewe- } \\
\text { berekonstruktion mittels externer Gewebeexpansion }\end{array}$ & Allgemein- u. Viszeralchirurgie \\
\hline Aachen & DFG Sachbeihilfe & $\begin{array}{l}\text { Die Rolle der Makrophagen migrationsinhibierenden Fak- } \\
\text { tor (MIF)-Proteinfamilie in Wundheilung und Übergewicht }\end{array}$ & Allgemein- u. Viszeralchirurgie & 2013-2017 \\
\hline Berlin & DFG Sachbeihilfe & $\begin{array}{l}\text { Antike Chirurgie zwischen Abend- und Morgenland: } \\
\text { Die Chirurgischen Handbücher (cheirourgoúmena) des } \\
\text { Leonides, Archigenes, Heliodoros und Antyllos - eine } \\
\text { medizinisch-philologische Bestandsaufnahme anhand } \\
\text { der überlieferten Fragmente. }\end{array}$ & Wissenschaftsgeschichte \\
\hline Berlin & DFG Sachbeihilfe & $\begin{array}{l}\text { Griechische Erstedition der Libri medicinales, Bücher 10 } \\
\text { und 14, des Aëtios von Amida (6. Jh. n. Chr.) samt deut- } \\
\text { scher Übersetzung, medizinhistorisch-philologischem } \\
\text { Kommentar und Quellenanalyse. }\end{array}$ & $\begin{array}{l}\text { Wissenschaftsgeschichte } \\
\text { Klassische Philologie }\end{array}$ \\
\hline Bochum & DFG Sachbeihilfe & $\begin{array}{l}\text { Mesenschymale Stammzellen und Wnt-Proteine in der } \\
\text { Therapie der posttraumatischen Osteomyelitis }\end{array}$ & Unfallchirurgie und Orthopädie \\
\hline Bochum & DFG Sachbeihilfe & $\begin{array}{l}\text { Untersuchungen zur Optimierung der Frakturheilung bei } \\
\text { Typ 2 diabetogener Stowechsellage mittels Wachstums- } \\
\text { faktoren und Stammzellen aus Fettgewebe }\end{array}$ & Unfallchirurgie und Orthopädie \\
\hline & seit 2016 & \\
\hline
\end{tabular}


- Tab. 1 Fortsetzung.

\begin{tabular}{|c|c|c|c|c|}
\hline Uniklinik & Art der Förderung & Projekttitel & Zuordnung & Zeitraum \\
\hline Bochum & $\begin{array}{l}\text { DFG Schwerpunkt- } \\
\text { programme }\end{array}$ & $\begin{array}{l}\text { Diagnose von Brandwunden mit mikrowellenbasierter } \\
\text { Nahfeldbildgebung }\end{array}$ & $\begin{array}{l}\text { Nachrichten- und Hochfrequenztechnik, } \\
\text { Kommunikationstechnik und -netze, } \\
\text { Theoretische Elektrotechnik }\end{array}$ & seit 2015 \\
\hline Bochum & $\begin{array}{l}\text { BMWi ZIM Sach- } \\
\text { beihilfe }\end{array}$ & $\begin{array}{l}\text { Entwicklung, Analyse, Zytokompatibilitätstests und } \\
\text { Darstellung der hydrophob-oleophoben Eigenschaften } \\
\text { innovativ beschichteter chirurgischer Instrumente }\end{array}$ & Materialwissenschaften & seit 2016 \\
\hline Bochum & $\begin{array}{l}\text { BMWi ZIM Sach- } \\
\text { beihilfe }\end{array}$ & $\begin{array}{l}\text { Entwicklung eines Implantats am distalen Radius, das } \\
\text { durch eine haftungsarme, auf plasma-elektrolytischer } \\
\text { Oxidation basierender Beschichtung und eine neuartige, } \\
\text { geometrisch durchbrochene Auflagefläche Sehnenver- } \\
\text { klebungen und Knochenintegration verhindert }\end{array}$ & Materialwissenschaften & seit 2016 \\
\hline Bochum & $\begin{array}{l}\text { BMWi ZIM Sach- } \\
\text { beihilfe }\end{array}$ & $\begin{array}{l}\text { Entwicklung eines Korrosionsprüfstandes für die Prädik- } \\
\text { tion des Verhaltens von Metalllegierungen in vivo }\end{array}$ & Materialwissenschaften & seit 2018 \\
\hline Erlangen & DFG Sachbeihilfe & $\begin{array}{l}\text { Tissue Engineering von axial vaskularisiertem Skelett- } \\
\text { muskelgewebe auf funktionellen Nanofaser-Scaffolds im } \\
\text { Tiermodell der Ratte }\end{array}$ & Unfallchirurgie und Orthopädie & 2013-2018 \\
\hline Erlangen & DFG Transregios & $\begin{array}{l}\text { Biofabrikation zellularisierter und im AV Loop vaskula- } \\
\text { risierter Gewebecontainer für die Transplantation wirk- } \\
\text { stoffproduzierender Zellen }\end{array}$ & $\begin{array}{l}\text { Hämatologie, Onkologie, Transfusions- } \\
\text { medizin, Biomaterialien; Verbundprojekt } \\
\text { mit Teilprojekt der Plastischen Chirurgie }\end{array}$ & seit 2018 \\
\hline Erlangen & DFG Transregios & $\begin{array}{l}\text { Analyse von Tumor Dormancy und Progression in bio- } \\
\text { fabrizierten und in vivo vaskularisierten 3D Modellen }\end{array}$ & $\begin{array}{l}\text { Zellbiologie, Biomaterialien; Verbund- } \\
\text { projekt mit Teilprojekt der Plastischen } \\
\text { Chirurgie }\end{array}$ & seit 2018 \\
\hline Freiburg & DFG Sachbeihilfe & $\begin{array}{l}\text { C-reaktives Protein (CRP) als pathogenetischer Faktor bei } \\
\text { Entzündung und Ischämie/ Reperfusion: Therapeutische } \\
\text { Implikationen }\end{array}$ & Allgemein - u. Viszeralchirurgie & seit 2010 \\
\hline Freiburg & DFG Sachbeihilfe & $\begin{array}{l}\text { Die Interaktion von C-reaktivem Protein (CRP) mit dem } \\
\text { Komplementsystem in der Pathogenese, Diagnostik und } \\
\text { Therapie der posttraumatischen Entzündungsantwort }\end{array}$ & Unfallchirurgie und Orthopädie & seit 2016 \\
\hline Freiburg & DFG Sachbeihilfe & Bioprinting von vaskularisiertem Knochenersatzgewebe & $\begin{array}{l}\text { Unfallchirurgie und Orthopädie, } \\
\text { Bioverfahrenstechnik }\end{array}$ & seit 2015 \\
\hline Freiburg & DFG Sachbeihilfe & $\begin{array}{l}\text { Präklinische in vitro Validierung und in vivo Evaluation } \\
\text { eines neuartigen Biohybrid-Gradienten-Schichtsystem- } \\
\text { Vlieses als Trägermatrix für eine vaskulogene Komponen- } \\
\text { te zur Versorgung epithelialer Weichgewebsdefekte }\end{array}$ & $\begin{array}{l}\text { Zahnheilkunde; Mund-, Kiefer- und } \\
\text { Gesichtschirurgie }\end{array}$ & 2014-2017 \\
\hline Freiburg & $\begin{array}{l}\text { DFG Heisenberg } \\
\text { Professur }\end{array}$ & & Allgemein - u. Viszeralchirurgie & seit 2016 \\
\hline Freiburg & BMBF & $\begin{array}{l}\text { Entwicklung und Erforschung einer Plattform für das } \\
\text { 3D-Bio-Printing von künstlichem Gewebe. Teilprojekt: } \\
\text { Charakterisierung und in vivo Experimente an Knochen } \\
\text { und Knorpel. }\end{array}$ & $\begin{array}{l}\text { Verbundprojekt mit Teilprojekt der Plas- } \\
\text { tischen Chirurgie }\end{array}$ & seit 2017 \\
\hline Freiburg & DFG Sachbeihilfe & $\begin{array}{l}\text { Zirkulierende Tumor DNA als nicht-invasiver diagnosti- } \\
\text { scher Biomarker bei Weichteilsarkomen }\end{array}$ & Allgemein - u. Viszeralchirurgie & seit 2018 \\
\hline Hannover & $\begin{array}{l}\text { DFG Forscher- } \\
\text { gruppen }\end{array}$ & Zytokompatibilitäts- und Bioaktivitätsprüfung in vitro & Biomaterialien & seit 2015 \\
\hline Lübeck & BMBF & Smart Scar Care & $\begin{array}{l}\text { Verbundprojekt mit Teilvorhaben der } \\
\text { Plastischen Chirurgie }\end{array}$ & 2016-2019 \\
\hline Magdeburg & BMWi & $\begin{array}{l}\text { Effects of weightlessness on the transcription behavior of } \\
\text { normal and malignant human cells }\end{array}$ & Plastische Chirurgie & $2016-2020$ \\
\hline Magdeburg & ESA-CORA-GBF (EU) & $\begin{array}{l}\text { Microgravitiy-dependent changes of gene and protein } \\
\text { expression in human normal and malignant thyroid cells }\end{array}$ & Plastische Chirurgie & 2014-2019 \\
\hline $\begin{array}{l}\text { Regens- } \\
\text { burg }\end{array}$ & DFG Sachbeihilfe & $\begin{array}{l}\text { Molekulargenetische, histologische, immunhistochemi- } \\
\text { sche und funktionelle Charakterisierung von Fettgewebe } \\
\text { bei Patienten mit Multipler Symmetrischer Lipomatose }\end{array}$ & Humangenetik, Dermatologie & seit 2015 \\
\hline $\begin{array}{l}\text { TU } \\
\text { München }\end{array}$ & BMWi & $\begin{array}{l}\text { Lymphknotenregeneration- Entwicklung von Bioartifiziellen } \\
\text { Lymphknoten zur Therapie des chronischen Lymphödems }\end{array}$ & Regernerative Medizin & 2016-2018 \\
\hline Würzburg & DFG Großgeräte & Mikrozirkulation bei perforatorbasiertem Gewebetransfer & Unfallchirurgie und Orthopädie & seit 2017 \\
\hline
\end{tabular}


- Tab. 2 Übersicht über die bewilligte Forschungsförderung öffentlicher, nicht-öffentlicher, industrieller oder anderer Institute (exklusive DFG, BMBF, BMWi, EU) nach Standorten.

\begin{tabular}{|c|c|c|c|c|}
\hline Uniklinik & Art der Förderung & Projekttitel & Zuordnung & Zeitraum \\
\hline Bochum & DGUV Sachbeihilfe & $\begin{array}{l}\text { Charakterisierung der systemischen Kinetik von Effek- } \\
\text { tormolekülen der epithelialen Immunantwort und } \\
\text { mikroRNA bei schweren Brandverletzungen und Sepsis }\end{array}$ & Plastische Chirurgie & seit 2017 \\
\hline Dresden & $\begin{array}{l}\text { AO Foundation; Forscher- } \\
\text { gruppe }\end{array}$ & $\begin{array}{l}\text { Preclinical evalation of a tissue-engineered vasculari- } \\
\text { zed bone implant }\end{array}$ & $\begin{array}{l}\text { Unfallchirurgie und Orthopädie; } \\
\text { Kooperationspartner Abteilung für } \\
\text { Plastische und Handchirurgie UKD }\end{array}$ & 2018 \\
\hline Erlangen & $\begin{array}{l}\text { Manfred Roth Stiftung/ } \\
\text { Forschungsstiftung } \\
\text { Medizin, Sachbeihilfe }\end{array}$ & $\begin{array}{l}\text { Entwicklung eines autonomen Lymphgefäßsystems } \\
\text { zum Einsatz in der regenerativen Medizin und als } \\
\text { Modellsystem zur Lymphangiogenese- und Antilym- } \\
\text { phangiogeneseforschung }\end{array}$ & Plastische Chirurgie & bis 2019 \\
\hline Erlangen & Staedler, Sachbeihilfe & $\begin{array}{l}\text { Entwicklung einer individualisierten Therapie chro- } \\
\text { nischer Wunden unter Anwendung körpereigener } \\
\text { Stammzellen }\end{array}$ & Plastische Chirurgie & 2018-2019 \\
\hline Erlangen & KCI USA Inc., Sachbeihilfe & $\begin{array}{l}\text { The next step: Innovative therapeutic approaches to } \\
\text { optimize the wound healing by combining Celluto- } \\
\text { meTM epidermal grafts, adipose-derived stem cells } \\
\text { and growth factors in hard to heal wounds }\end{array}$ & Plastische Chirurgie & 2016-2018 \\
\hline Erlangen & $\begin{array}{l}\text { Manfred- Roth- Stiftung, } \\
\text { Sachbeihilfe }\end{array}$ & $\begin{array}{l}\text { Den Blutgefäßen beim Wachsen zuschauen - } \\
\text { Gewebszüchtung im AV-Loop-Modell unter dem } \\
\text { Intravitalmikroskop }\end{array}$ & Plastische Chirurgie & 2018-2019 \\
\hline Freiburg & $\begin{array}{l}\text { Robert-Pfleger Stiftung } \\
\text { Sachbeihilfe }\end{array}$ & $\begin{array}{l}\text { Modulation der Immunogenität und des regenerativen } \\
\text { Potenzials fötaler endothelialer Progenitorzellen } \\
\text { durch CRISPR/Cas9-vermittelte Inaktivierung der MHC } \\
\text { Expression }\end{array}$ & & seit 2018 \\
\hline $\begin{array}{l}\text { Halle/ } \\
\text { Leipzig }\end{array}$ & $\begin{array}{l}\text { Else-Kröner-Fresenius-Stif- } \\
\text { tung; Forschergruppe }\end{array}$ & $\begin{array}{l}\text { Modulation der Adipozytenvitalität nach autologer } \\
\text { Fettgewebstransplantation durch repetitive Leptin- } \\
\text { applikationen }\end{array}$ & Plastische Chirurgie & 12 Monate \\
\hline $\begin{array}{l}\text { Halle/ } \\
\text { Leipzig }\end{array}$ & $\begin{array}{l}\text { DGUV und Firma Bauer- } \\
\text { feind, Sachbeihilfe }\end{array}$ & $\begin{array}{l}\text { Mikroskopische Untersuchungen der Innervation des } \\
\text { Handgelenkes, des distalen Radioulnargelenkes, der } \\
\text { Membrana interossea antebrachii und der Ellenbo- } \\
\text { genbänder an humanen Leichenpräparaten }\end{array}$ & Handchirurgie & $\begin{array}{l}\text { bis } \\
31.05 .2019\end{array}$ \\
\hline Hannover & $\begin{array}{l}\text { MWK Niedersachsen/VW } \\
\text { Stiftung; Forschungsver- } \\
\text { bund }\end{array}$ & Biofabrication, Modul 1 Tissue Engineering & & 2016-2018 \\
\hline Hannover & $\begin{array}{l}\text { Fritz Behrens Stiftung } \\
\text { Sachbeihilfe }\end{array}$ & $\begin{array}{l}\text { Herstellung natürlicher Implantate mit sehr niedriger } \\
\text { Immunogenität }\end{array}$ & & 2016-2019 \\
\hline Heidelberg & DGUV Sachbeihilfe & $\begin{array}{l}\text { Transkulturelle Adaptation und Validierung des Burn- } \\
\text { Specific-Health-Scale brief }\end{array}$ & $\begin{array}{l}\text { Plastische Chirurgie BGU Ludwigs- } \\
\text { hafen }\end{array}$ & 2 Jahre \\
\hline Heidelberg & DGUV Sachbeihilfe & $\begin{array}{l}\text { Evaluation des ICF-orientierten „Rehabilitationskon- } \\
\text { zepts thermische Verletzungen }\end{array}$ & $\begin{array}{l}\text { Plastische Chirurgie BGU Ludwigs- } \\
\text { hafen }\end{array}$ & 4 Jahre \\
\hline Heidelberg & DGUV Sachbeihilfe & $\begin{array}{l}\text { Funktioneller Gewebeersatz mit in vivo gezüchteten } \\
\text { vaskularisierten Bindegewebslappenplastiken im } \\
\text { Großtiermodell }\end{array}$ & $\begin{array}{l}\text { Plastische Chirurgie BGU Ludwigs- } \\
\text { hafen }\end{array}$ & 3 Jahre \\
\hline Heidelberg & $\begin{array}{l}\text { Alexander von Humboldt } \\
\text { Stiftung Sachbeihilfe }\end{array}$ & $\begin{array}{l}\text { Regeneration von radiogenen Knochendefekten nach } \\
\text { Malignomerkrankung durch axial vaskularisierte } \\
\text { Tissue Engineering Konstrukte }\end{array}$ & $\begin{array}{l}\text { Plastische Chirurgie BGU Ludwigs- } \\
\text { hafen }\end{array}$ & 2 Jahre \\
\hline Heidelberg & $\begin{array}{l}\text { Hopp-Stiftung Walldorf } \\
\text { Forschergruppe }\end{array}$ & $\begin{array}{l}\text { Mesodermale Regeneration mit mesenchymalen } \\
\text { Stammzellen aus Fettgewebe }\end{array}$ & Plastische Chirurgie & $\begin{array}{l}\text { bis } \\
\text { Ende } 2018\end{array}$ \\
\hline $\begin{array}{l}\text { Köln } \\
\text { Merheim }\end{array}$ & $\begin{array}{l}\text { Else-Kröner-Fresenius- } \\
\text { Stiftung }\end{array}$ & $\begin{array}{l}\text { Charakterisierung der zytoprotektiven und wundhei- } \\
\text { lungsfördernden Wirkung des Growth/Differentiation } \\
\text { Factor } 5 \text { (GDF-5) in einem in vitro Zellkulturmodell } \\
\text { sowie ex vivo in einem Verbrennungsmodell der } \\
\text { menschlichen Haut }\end{array}$ & Plastische Chirurgie & 2 Jahre \\
\hline Regensburg & Manfred Roth Stiftung & $\begin{array}{l}\text { Optimierung der Transplantatkonservierung durch } \\
\text { unidirektionale extrakorporale Perfusion von Myokut- } \\
\text { anlappen im Großtier-Replantations-Modell (Schwein) }\end{array}$ & Plastische Chirurgie & seit 2017 \\
\hline
\end{tabular}


> Tab. 2 Fortsetzung.

\begin{tabular}{|c|c|c|c|c|}
\hline Uniklinik & Art der Förderung & Projekttitel & Zuordnung & Zeitraum \\
\hline Regensburg & $\begin{array}{l}\text { Virtuelle Hochschule } \\
\text { Bayern }\end{array}$ & Ambulante Notfallversorgung von Handverletzungen & Handchirurgie & 2016-2018 \\
\hline Regensburg & $\begin{array}{l}\text { Virtuelle Hochschule } \\
\text { Bayern }\end{array}$ & $\begin{array}{l}\text { Rekonstruktive Verfahren zur Wiederherstellung der } \\
\text { weiblichen Brust }\end{array}$ & Plastische Chirurgie & 2018 \\
\hline TU München & $\begin{array}{l}\text { ASC SKIN THERAPEUTICS } \\
\text { LTD }\end{array}$ & $\begin{array}{l}\text { Analysis of the regenerative mechanism of Momen- } \\
\text { tum BioniCs } ® \text { utilizing an in vivo porcine wound and } \\
\text { scar model }\end{array}$ & Plastische Chirurgie & seit 2017 \\
\hline TU München & $\begin{array}{l}\text { ASC SKIN THERAPEUTICS } \\
\text { LTD }\end{array}$ & $\begin{array}{l}\text { A multicenter, double blind, randomized, placebo } \\
\text { controlled phase Ila study to assess the safety and } \\
\text { efficacy of Momentum BioniCs hydrogel for topical } \\
\text { treatment of grade II scald injuries in children } \\
\text { between } 9 \text { months and } 5 \text { years of age }\end{array}$ & Plastische Chirurgie & 2017-2020 \\
\hline TU München & $\begin{array}{l}\text { ASC SKIN THERAPEUTICS } \\
\text { LTD }\end{array}$ & $\begin{array}{l}\text { To assess the safety and efficacy of Momentum Bio- } \\
\text { niCs hydrogel for topical treatment of split thickness } \\
\text { skin graft donor sites in diabetic patients }\end{array}$ & Plastische Chirurgie & 2017-2020 \\
\hline TU München & $\begin{array}{l}\text { Bayrische Forschungs- } \\
\text { stiftung }\end{array}$ & $\begin{array}{l}\text { Injizierbare Hightech-Füllmaterialien zur Gewebeaug- } \\
\text { mentation auf Basis von Spinnenseide }\end{array}$ & Plastische Chirurgie & 2016-2018 \\
\hline TU München & Lipedema Foundation & $\begin{array}{l}\text { Highly Parallel Genome-wide Expression Profiling of } \\
\text { Lipedema Tissue on a Single Cell Level using Nanoliter } \\
\text { Droplets }\end{array}$ & Plastische Chirurgie & 2017-2018 \\
\hline TU München & Tomorrowlabs GmbH & HIF-1Alpha Modulation zur Gewebsregeneration & Plastische Chirurgie & $2017-2020$ \\
\hline TU München & $\begin{array}{l}\text { ProFIT-Investitionsbank; } \\
\text { BIOTRONIK SE }\end{array}$ & $\begin{array}{l}\text { 4D CAE-Herzmodellerstellung zur Optimierung von } \\
\text { Mess- und Therapiegeräten des Herzrhythmusma- } \\
\text { nagements }\end{array}$ & Plastische Chirurgie & 2017-2019 \\
\hline TU München & $\begin{array}{l}\text { Kommission für Kli- } \\
\text { nische Forschung der TU } \\
\text { München }\end{array}$ & $\begin{array}{l}\text { Optimaler zellunterstützter Lipotransfer zur Weich- } \\
\text { teilregeneration }\end{array}$ & Plastische Chirurgie & 2018-2019 \\
\hline Tübingen & $\begin{array}{l}\text { TÜFF der Universität } \\
\text { Tübingen Forschergruppe }\end{array}$ & $\begin{array}{l}\text { Sind Wachstumsfaktoren die Lösung zur beschleunig- } \\
\text { ten Wundheilung? }\end{array}$ & Plastische Chirurgie & 12 Monate \\
\hline
\end{tabular}

zu „Herz-, Thorax-, Gefäßchirurgie“, „Plastische Chirurgie“, „Orthopädie und Unfallchirurgie“ bzw. „sonstige“ unterteilt. Da Anträge häufig aus Kliniken für Herz-, Thorax- und Gefäßchirurgie ausgingen, war für eine weitere Unterteilung in die seperaten Fachgebiete die Zuordnung bzw. der klinische Schwerpunkt des Antragstellers (Thorax- oder Gefäßchirurgie) bzw. der Titel des Projektes entscheidend. Nur Projekte aus den Jahren 2017/2018 wurden gewertet.

\section{Ergebnisse}

Für die Plastische Chirurgie wurden im Jahr 2017/2018 an 12 universitären Standorten Fördermittel von DFG, BMBF, BMWi oder EU beantragt. Dabei gingen von einer Institution zwischen 1 und 8 Anträge aus.

Insgesamt konnten im GEPRIS und nach Auswertung der Angaben in unserer Internetplattform 34 Förderanträge der öffentlichen Förderinstitute DFG, BMBF, BMWi und EU identifiziert werden. Davon waren 70,6\% (24) DFG, 14,7\% (5) BMWi, 11,8\% (4) BMBF und 2,9\% (1) EU Anträge. Wie aus \ Tab. 1 zu entnehmen waren 79,4\% (27) der Anträge zum Zeitpunkt der Datenerhebung bereits bewilligt, davon 70,4\% (19) durch DFG, 7,4\% (2) durch BMBF, 18,5\% (5) durch BMWi und 3,7\% (1) durch EU. 8,8\% (3) der Anträge befanden sich während dieser Auswertung noch in Begutachtung, davon waren zwei beim BMBF und ein Antrag bei der DFG einge- reicht. 11,8\% (4) der Anträge wurden abgelehnt, diese waren alle bei der DFG eingereicht worden.

$22,2 \%$ (6) der genannten bewilligten Anträge waren der „Unfallchirurgie und Orthopädie“, 18,5\% (5) waren der „Allgemeinund Viszeralchirurgie“, 14,8\% (4) waren „Materialwissenschaften“ oder „Biomaterialien“ zugeordnet, 37,0\% (10) waren sonstigen oder mehreren Fachgebieten zugeordnet. Lediglich 7,4\% (2) der Anträge bei DFG, BMBF, BMWi und EU waren der Plastischen Chirurgie zugeordnet.

In 68,4\% (13) der Fälle wurden die bewilligten DFG Anträge als Sachbeihilfe eingestuft.

10,5\% (2) der DFG Anträge waren Transregios. Je 1 Antrag war Schwerpunktprogrammen, Forschergruppen, Heisenberg-Professuren oder Großgeräten zugeordnet.

Neben den Anträgen bei oben genannten öffentlichen Förderinstitutionen, sind der Vollständigkeit halber in \ Tab. 2 auf der Internetplattform angegebene Forschungsanträge anderer öffentlicher, nicht-öffentlicher, oder industrieller Institute dargestellt. Neben den oben genannten Anträgen von DFG, BMBF, BMWi und EU wurden 35 sonstige Anträge angegeben. Von diesen waren 82,9\% (29) bewilligt. Von den bewilligten Anträgen waren 17,2\% (5) DGUV Anträge, 10,3\% (3) Anträge bei der Manfred- Roth- Stiftung und 6,9\% (2) Anträge der Else-Kröner-Fresenius-Stiftung. Interessanterweise 
- Tab. 3 Übersicht über die bewilligte DFG Forschungsförderung und fachliche Zuordnung der bewilligten Anträge von Thorax-, Gefäß- und Plastischer Chirurgie nach GEPRIS.

\begin{tabular}{|l|l|l|l|l|}
\hline & fachliche Zuordnung & $\begin{array}{l}\text { Herz-, Thorax-, Gefäß- } \\
\text { chirurgie }\end{array}$ & $\begin{array}{l}\text { Orthopädie und Unfall- } \\
\text { chirurgie }\end{array}$ & Sonstige \\
\hline Antragsteller & & & & 5 \\
\hline Thoraxchirurgie & 4 & 1 & 5 & 8 \\
\hline Gefäßchirurgie & 2 & 6 & 13 & 19 \\
\hline Plastische Chirurgie & 1 & & 6 \\
\hline
\end{tabular}

konnten hier 82,8\% (24) der bewilligten Anträge der Plastischen Chirurgie zugeordnet.

- Tab. 3 zeigt den Vergleich der bewilligten DFG Anträge der kleineren Fachgebiete Thorax- und Gefäßchirurgie im Vergleich zur Plastischen Chirurgie und der jeweiligen fachlichen Zuordnung. Von den kleineren chirurgischen Fachgebieten wurden dem GEPRIS folgend von Thoraxchirurgie und Gefäßchirurgie jeweils 9 bzw. 8 DFG Anträge bewilligt. Davon waren für die Thoraxchirurgie 44\% (4) und für die Gefäßchirurgie 25 \% (2) der Anträge dem eigens vorgesehenen Fachkollegium der „Herz-, Thorax-, Gefäßchirurgie“ zugeordnet. Im Vergleich dazu konnten 19 bewilligte Anträge der Plastischen Chirurgie identifiziert werden. Lediglich 32\% (6) der 19 plastisch-chirurgischen Anträge waren dem vom DFG vorgesehenen Fachkollegium der „Orthopädie und Unfallchirurgie“ zugeordnet.

\section{Diskussion}

Forschungsförderung spielt eine essentielle Rolle in der Bewertung der akademischen Leistung eines Fachgebiets. Die Wertigkeit eines Fachgebiets wird nicht zuletzt besonders anhand der wissenschaftlichen Exzellenz und den in Fachjournalen publizierten Arbeiten beurteilt. Aus diesem Grund ist die Bekanntmachung der zunehmenden Bandbreite der Forschungsaktivität von großem öffentlichen Interesse. Die regelmäßige Auswertung der Forschungsaktivität und die damit erreichte Sichtbarkeit ist das Ziel dieses Forschungsberichts der DGPRÄC. Auch Arbeiten wie die von Alawi et al., in der eine systematische Analyse der Publikationsleistung der deutschen, universitären Plastischen Chirurgie durchgeführt wurde, sind in dieser Hinsicht von substanzieller Bedeutung [7].

Im Vergleich zum Forschungsförderungsbericht 2015/2016 konnte die Anzahl an bewilligten DFG Anträgen nahezu wiederholt werden (vgl. 21 bewilligte Anträge 2015/16 vs. 19 bewilligte Anträge 2017/18). Insgesamt konnte unser Fachgebiet die Anzahl der bewilligten Anträge von DFG, BMBF, BMWi und EU im Jahr 2017/18 von 23 auf 27 steigern. Waren im Jahr 2015/2016 je 2 Anträge bewilligte EU und BMBF Forschungsförderungen, wurden 2017/2018 1 EU, 2 BMBF und 5 BMWi Anträge bewilligt [1].

Allerdings wird das Fachgebiet Plastische Chirurgie bisher noch nicht in der Fächerstruktur der DFG abgebildet. Stattdessen wird eine Zuordnung zur Orthopädie und Unfallchirurgie getroffen. Das Fachkollegium 205-27 soll trotz aller Bemühungen der Fachgesellschaft DGPRÄC um eine eigene Kategorie zukünftig in „Orthopädie, Unfallchirurgie, rekonstruktive Chirurgie“ umbenannt werden und damit unser seit 1993 nunmehr 25 Jahre eigenständiges
Fachgebiet weiterhin explizit unerwähnt bleiben. Obwohl das Forschungsaufkommen in der Plastischen Chirurgie nachweislich das anderer kleinerer chirurgischer Fachgebiete, wie beispielsweise der Thorax- oder Gefäßchirurgie, zahlenmäßig übertrifft und zumindest nominell gleichwertig ist, hat dies zu diesem Zeitpunkt noch keine Anpassung der Fächerstruktur bewirken können. Damit wird aus unserer Perspektive die sich dem medizinischen Forstschritt in der Chirurgie stetig wandelnde Fächerstruktur an Universitätskliniken nicht ausreichend gewürdigt.

In der Zuordnung zu „Orthopädie, Unfallchirurgie und rekonstruktive Chirurgie“ ist unser Fachgebiet aus Sicht der beteiligten Forscher nicht ausreichend klar definiert. Unsere Auswertung zeigt, dass $68 \%$ unserer bewilligten DFG Anträge im Jahr 2017/18 anderen Fachgebieten, wie beispielsweise der „Allgemein- und Viszeralchirurgie“ zugeordnet und damit meist überhaupt nicht sichtbar als Forschungsleitung der Plastischen Chirurgie waren.

Für unsere Fachgesellschaft besteht über ein Vorschlagsrecht im Jahr 2019 die Möglichkeit, Kandidatinnen und Kandidaten aus dem Bereich Plastische Chirurgie für die kommende Amtsperiode der Fachkollegien (2020-2023) vorzuschlagen. [8] Eine Wahl könnte dann aber nur eine Beteiligung innerhalb des großen - für die Plastische Chirurgie nicht repräsentativen - Fachkollegiums erfolgen.

\section{Ausblick}

Ziel der Forschungsberichte der DGPRÄC ist es weiterhin die Forschung unseres Fachgebiets Plastische Chirurgie öffentlich zu machen, aber auch die mit öffentlichen Mitteln ermöglichten Leistungen transparent zu machen. Die erkennbare hohe qualitative Produktivität und Innovation kann auch dann als Entscheidungshilfe für die weitere akademische Weiterentwicklung in den medizinischen Fakultäten dienen.

\section{Interessenkonflikt}

Die Autoren geben an, dass kein Interessenkonflikt besteht.

\section{Literatur}

[1] Giunta RE, Horch RE, Prantl L et al. [Registry Research Funding of the German Society of Plastic, Reconstructive and Aesthetic Surgeons 
(DGPRAC) and Research Funding Report 2015/2016]. Handchir Mikrochir Plast Chir 2016; 48: 370-373

[2] Neumeister MW. Can a Plastic Surgeon be a Department Chairman?.... Really? Handchir Mikrochir Plast Chir 2016; 48: 69-72

[3] Schubert CD, Haertnagl F, Elsner J et al. [Are autonomous departments better research institutions? Influence of independence on publication performance and study-types in academic Plastic Surgery]. Handchir Mikrochir Plast Chir 2018; 50: 277-283

[4] Liu P, Singh M, Eriksson E. Academic Status of Plastic Surgery in the United States and the Relevance of Independence. Handchir Mikrochir Plast Chir 2016; 48: 65-68

[5] Giunta RE, Machens HG. [Science and research in academic plastic surgery in Germany]. Handchir Mikrochir Plast Chir 2009; 41: 359-363

[6] http://gepris.dfg.de/.

[7] Alawi SA, Bohr S, Stromps JP et al. [Research Assessment in Plastic and Reconstructive Surgery on a National Level: A 5-Years Systematic Review of Research Activity in German University Hospitals]. Handchir Mikrochir Plast Chir 2016; 48: 73-77

[8] http://www.dfg.de/dfg_profil/gremien/fachkollegien/fk_wahl2019/ index.jsp. 